\title{
Medicine partnerships
}

\section{J Cramer}

Heart 2003;89(Suppl II):ii 19-ii2 1

Partial medication compliance, where patients do not take enough of their prescribed medicine to achieve adequate outcomes, is common. Research using electronic monitoring to assess compliance has shown that people take approximately $75 \%$ of doses as prescribed, irrespective of the condition being treated or its severity. Erratic compliance often leads to discontinuation of therapy, as treatment is perceived to be ineffective. Compliance decreases as frequency of dosing increases. Inadequate compliance and treatment persistence results in poor outcomes, despite the best efforts of the medical team. It is important to develop and implement a strategy to improve compliance. Simple steps that can be taken include helping patients to select "cues" to remind them to take their tablets, use of dose reminder boxes, and visual feedback of compliance data from electronic monitors.

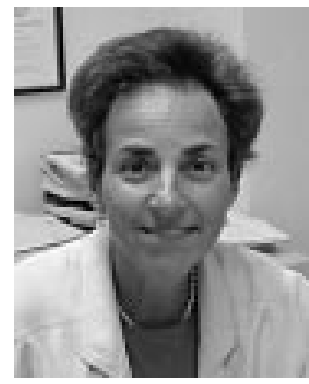

$M$ edication compliance can be defined as the extent to which the patient follows mutually agreeable instructions, or the extent to which actual drug taking behaviour matches the prescribed regimen. Other terms used to describe the same concept include adherence, precision, and concordance. Noncompliance, which is probably relatively uncommon, occurs when the patient has a lack of belief in the diagnosis and the need to take medication.

Realistically, it is not fair to think that patients must (or will) take every tablet, every day. What is important is outcome measurement-for example, achieving good blood pressure control. It is likely that only half of patients take enough medication to be called full (adequate) compliers. Partial (inadequate) compliance is common. It can relate to an occasional missed dose, an occasional extra dose, erratic dosing, or a consistent pattern but not as prescribed. Treatment persistence is another aspect of compliance, with a focus on long term continuation of treatment. Nonpersistence refers to the situation when patients feel they no longer need medication and discontinue treatment without medical recommendation.

Partial compliers are well intentioned patients who fill their prescription and return to the physician for visits but who, in the context of cardiovascular disease, do not take enough medication to achieve good blood pressure, lipid concentrations or glucose control. These patients are difficult for clinicians to manage; they may lead the prescriber to question whether the right diagnosis has been made and the appropriate medication has been selected. Partial compliance typically leads to prescribing of higher doses, or a different drug, while the problem is actually that
Connecticut, USA:

joyce.cramer@yale.edu the medicine has not been taken. It is essential to recognise that poor compliance may explain a patient's inadequate response to treatment.

Typical reasons for partial compliance and nonpersistence are lack of planning, forgetfulness, altered daily schedule, confusion about instructions, lack of efficacy (which in turn could be related to inadequate compliance), adverse effects, and cost.

Many attempts have been made to predict compliance but this is difficult. It is not related to sociodemographic variables or to psychological profiles, such as intelligence or memory. Questionnaires have been developed that are claimed to be able to predict compliance but these are highly biased. Doctors or families are also poor at predicting compliance. The best predictor is the individual's own past performance.

\section{MEASURING COMPLIANCE}

Medication compliance, both in research and in everyday practice, can be assessed by continuous electronic monitoring, using a standard tablet bottle with a microprocessor in the cap that records the date and time whenever the bottle is opened. Bottle caps may be plain or may have a display that shows the patient how many times that day the bottle has been opened. The use of these devices can help patients improve their medication compliance. Patients can associate dosing with control of their disorder and this helps with self management of their condition. People want to be empowered to take care of themselves and to make decisions in partnership with the doctor.

The use of electronic monitoring to understand how patients are actually taking their medication is now the gold standard in compliance research. A systematic review of the impact of dose regimen on medication compliance reviewed 76 papers that had used electronic monitors to assess rates of compliance and precision. ${ }^{1}$ Compliance was measured as the number of days that the prescribed number of tablets had been taken. The review confirmed the findings of an earlier report, ${ }^{2}$ with dose taking being inversely related to the prescribed number of doses per day. Compliance was $79 \%$ with once daily dosing, $69 \%$ with twice daily dosing, 65\% with three times daily dosing, and $51 \%$ with four times daily dosing. Compliance was significantly higher for once daily versus three times daily $(p=0.008)$, once daily versus four times daily $(p<0.001)$, and twice daily versus four times daily $(p=0.001)$ regimens.

One clear conclusion to draw from these data is that physicians should avoid prescribing four times daily regimens; while patients might be able to comply with such a regimen for an acute problem, few will be able to continue with it in the long term. For chronic illnesses, simplicity of regimen is extremely important. Taking all 


\begin{tabular}{|l|l|l|l|l|l|l|}
\hline Sun & Mon & Tue & Wed & Thu & Fri & Sat \\
\hline 0 & 1 & 2 & 2 & 2 & 1 & 0 \\
\hline 0 & 3 & 1 & 2 & 2 & 2 & 0 \\
\hline 0 & 1 & 2 & 2 & 1 & 1 & 1 \\
\hline 0 & 1 & 1 & 2 & 1 & 2 & 0 \\
\hline
\end{tabular}

Figure 1 Four week electronic dosing record for a patient prescribed a medication to be taken twice a day, showing erratic compliance on weekdays and neglect at the weekend.

regimens together, on average people take approximately $75 \%$ of medication as prescribed. ${ }^{1-3}$

Data from the electronic monitoring devices can be downloaded to a computer to give a visual representation of how often the patient took their medication, the number of hours between doses, and periods of missed dosing. As discussed further below, this can be used to work with patients to identify barriers to medication taking and to consider ways of overcoming them. Figure 1 shows such a "report card" on a patient who had been prescribed a twice daily regimen. It shows erratic compliance during the week and neglect at the weekend.

Dose precision-taking tablets within an appropriate time window-is far less accurate than dose taking. The systematic review $^{1}$ looked at a subset of 14 studies that assessed dose precision. Overall, mean dose timing compliance was 59\%, but the figure decreased with the number of daily doses. With a once daily schedule, $74 \%$ of patients took their tablets within a 24 hour window; with twice daily dosing $58 \%$ of patients took their tablets within 12 hours; the equivalent precision figures were $46 \%$ for three times daily dosing and $40 \%$ for four times daily dosing.

Disease severity does not affect compliance. Once daily dosing is not the answer, as shown by a survey of 13 studies involving patients taking once-a-day antihypertensive medication in which compliance ranged from $53-85 \%$, with a mean figure of $76 \% .^{3}$

Patient compliance with medicine taking can vary from day to day, from consistent to erratic dosing. For example, compliance has been shown to be significantly higher for the few days before and after a clinic visit and then to decrease again. ${ }^{4}$ Attention to dosing is enhanced in anticipation of a visit to the clinic when health behaviour is a prominent issue. The health behaviour lasts for a while, but then gradually falls off because of competing demands for time and interest.

It is important to note that lapses in drug taking can lead to risk of severe rebound effects, both from stopping the drug suddenly without a down titration and from restarting when the tablets are remembered again. Sudden stopping or starting of medication can lead to acute medical problems. Patients need to receive frequent and consistent feedback about the importance of taking medication every day and long term.

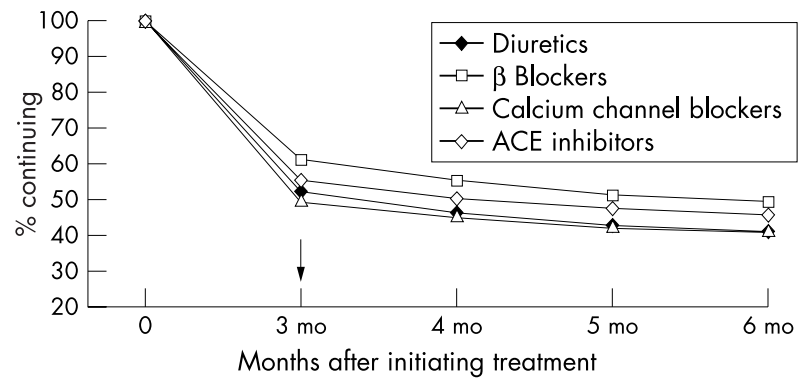

Figure 2 Poor treatment persistence with antihypertensive medications. Data from Jones et al. ${ }^{6}$

To summarise, compliance studies have shown that:

- people take (at best) approximately $75 \%$ of doses as prescribed

- erratic compliance often leads to discontinuation of therapy as treatment is perceived to be ineffective

- poor compliance and poor persistence with antihypertensive medication can contribute to the lack of adequate blood pressure control that is known to occur in more than two thirds of patients with hypertension.

\section{TREATMENT PERSISTENCE AND CLINICAL OUTCOMES}

A UK study ${ }^{6}$ showed that three months after starting treatment for hypertension, about $50 \%$ of patients had discontinued their medication (fig 2). By six months, 50-60\% of treatments had been changed or discontinued.

A retrospective, population based analysis of persistence with antihypertensive drug treatment in primary care practice was recently reported from Italy. ${ }^{7}$ The investigators looked at newly diagnosed patients prescribed various antihypertensive drugs and at their continued use of the drugs over the next 12 months. All prescriptions filled over the 12 months were used to define patients as continuers, switchers or discontinuers on the basis of their persistence with treatment. Less than a third of patients continued on their initial prescription while around $10 \%$ of patients switched treatment, but $65 \%$ of patients discontinued treatment. This happens routinelybetween $40-60 \%$ of patients will stop treatment and lose their treatment benefit in less than one year.

The relation between compliance and outcomes is demonstrated by data from the Lipid Research Clinics coronary primary prevention trial. ${ }^{8}$ In this seven year trial, patients who took the prescribed dosage of cholestyramine achieved better cholesterol reduction and overall cardiovascular risk reduction than those who took less than the prescribed dose. In the USA, these data are included in the package insert for cholestyramine to demonstrate to patients and health professionals the benefit of full compliance. It represents an important public health message.

Recent data from the PRISM (platelet receptor inhibition in ischaemic syndrome management) study show how withdrawal of statins affects event rates in patients admitted to

Table 1 Factors associated with compliance

\begin{tabular}{ll}
\hline Factors that correlate with compliance & Factors that do not correlate with compliance \\
\hline - Dosing regimen (less frequent regimens better) & - Sex \\
- Patient lifestyle routines & - Educational level \\
- Use of other medications & - Socioeconomic status \\
- Side effects (to some extent) & - Disease state \\
& - Disease severity \\
\hline
\end{tabular}


hospital with acute coronary symptoms. If the statin was withdrawn after admission, cardiac risk (death and non-fatal myocardial infarction) increased compared with patients who continued to receive statins $(p=0.005)$ and tended to be higher compared with patients who never received statins $(p=0.15)$, demonstrating the potential effect of noncompliance in a population at risk of cardiovascular events.

Inadequate compliance and persistence results in poor outcomes, despite the best efforts of the medical team. Given the scale of the problem, and its effect on everyday practice, it is essential to develop and implement a strategy to improve compliance.

\section{IMPROVING MEDICATION COMPLIANCE}

Given current knowledge about factors that are and are not correlated with compliance (table 1), there are some simple steps that can be taken to improve medication compliance.

The Medication Usage Skills for Effectiveness Program (MUSE-P) is a simple, office based teaching programme designed to enhance compliance. It takes less than five minutes on the first visit and two minutes at each follow up visit, and can be initiated by non-medical staff. As part of this programme, it is useful to suggest that the patient selects a dose reminder "cue". This can be any activity that they perform regularly that can be mentally associated with a scheduled dose. Basic cues are clock times, meal times, or daily rituals, such as shaving, walking the dog or watching the news. The aim is to develop a cue that reminds the patient to check that they have taken their medication.

Another tool to help the patient is the use of dose reminder boxes or bottles. The boxes are filled once a week and show clearly whether or not a dose has been taken. This helps patients to feel in control of their own disorder. Dose reminder bottles tell the patient how many times that day they have opened the bottle.

Visual feedback to the patient of compliance data from electronic monitoring is also useful. The patient can see the record of the doses they have taken. This feedback is non-judgmental. The doctor and patient can discuss the report, why compliance might have been a problem at particular times, and how it might be improved-for example, by selecting different cues. Electronic monitoring with feedback has proven very effective at improving compliance among patients with treatment refractory schizophrenia and intractable depression, ${ }^{10}$ with statistically significant improvement in overall compliance in the feedback group compared with the control group given usual care. Similarly positive results have been reported in patients with treatment refractory hypertension. ${ }^{11}$ With electronic monitoring and feedback, 34\% of patients with refractory hypertension attained satisfactory blood pressure control.

\section{CONCLUSION}

If hypertension, hyperlipidaemia, and diabetes are the three main risk factors for cardiovascular disease, poor treatment compliance and persistence can be considered to be the fourth risk factor. It is important to try to help patients who are partial compliers to develop better dosing habits. As well as choosing simple dose regimens to improve compliance, it can be useful for patients to select a dose reminder cue. Use of reminder boxes should also be encouraged and electronic monitors are helpful in providing feedback to the patient to enhance compliance.

Improved compliance brings benefit to the patient by improving treatment efficacy, benefit to the prescriber who no longer needs to keep switching medicines to try to find an effective treatment, and net benefit to the health care system by potentially leading to fewer cardiovascular events.

\section{Learning points}

- Once a prescriber has explained to the patient why medication is needed, focus on the importance of regular, long term dosing

- Help patients to develop cues to remind them to take their tablets

- Ask about compliance at every visit to keep the topic in the patient's mind and discuss any problems that the patient is having

\section{REFERENCES}

1 Claxton AJ, Cramer J, Pierce C. A systematic review of the associations between dose regimens and medication compliance. Clin Ther 2001;23:1296-310.

2 Cramer AJ, Mattson RH, Prevey ML, et al. How often is medication taken as prescribed? A novel assessment technique. JAMA 1989;261:3273-7

3 Cramer JA. Consequences of intermittent treatment for hypertension: the case for medication compliance and persistence. Am J Manag Care 1998:4:1563-8.

4 Cramer JA, Scheyer RD, Mattson RH. Compliance declines between clinic visits. Arch Int Med 1990;150:1509-10

5 Joint National Committee. The sixth report of the Joint National Committee on prevention, detection, evaluation and treatment of high blood pressure. Arch Intern Med 1997;157:2413-46.

6 Jones JK, Gorkin L, Lian JF, et al. Discontinuation of and changes in treatment after start of new courses of antihypertensive drugs: a study of a United Kingdom population. BM 1995;311:293-5.

7 Esposti LD, Esposti ED, Valpiani G, et al. A retrospective, population-based analysis of persistence with antihypertensive drug therapy in primary care practice in Italy. Clin Ther 2002;24:1347-57.

8 Lipids Research Clinics Program. The Lipid Research Clinics coronary primary prevention trial results. II: The relationship of reduction in incidence of coronary heart disease to cholesterol lowering. JAMA 1984:251:365-74.

9 Heeschen C, Hamm CW, Laufs U, et al, for the Platelet Receptor Inhibition in Ischemic Syndrome Management (PRISM) Investigators. Withdrawal of statins increases event rates in patients with acute coronary syndromes. Circulation 2002;105:1446-52.

10 Cramer JA, Rosenheck R. Enhancing medication compliance for people with serious mental illness. J Nervous Mental Dis 1999;187:53-4.

11 Burnier M, Schneider MP, Chiolero A, et al. Electronic compliance monitoring in resistant hypertension: the basis for rational therapeutic decisions. J Hypertens 2001;19:335-41.

\section{DISCUSSION}

Question: Can you explain why patients play a game? The doctor says "I am sorry, your blood pressure is not well controlled, we're going to have to increase the dose", but the patient knows that he is not taking the medication.

Ms Cramer: Most of the time people do not know they have missed their doses. They really believe that they take it properly.

Question: One concept that we use quite widely in the UK is that of intentional non-compliance, where people basically choose not to comply. It is not that they forget but they decide not to do it. To put compliance into context, I think it is important to consider to what extent we follow the recommendations of other professionals. Do you change your oil on time, do you check the tyres on your car every time you go to the garage? Do you think we have somewhat unrealistic expectations about the compliance that our patients should have?

Ms Cramer: Absolutely, and that is why I don't target 100\% but just aim to achieve the desired outcome. You may not change the oil in the car but you do keep enough petrol in there to keep you rolling. We call it intelligent noncompliance. The doctor will over-prescribe, and the patient says I am an older, smaller person, I don't need that much and I am going to cut my pill in half. Intelligent non-compliance is a different issue from partial compliance. 